\title{
A Tool for Promoting Responsible Antibiotic Prescribing across Settings and Sectors
}

\section{Anette Hulth ${ }^{1}$, Sonja Löfmark ${ }^{1}$, Jeff Andre ${ }^{2}$, Rachel Chorney ${ }^{2}$, Emily Cohn ${ }^{2}$, Moriah Ellen ${ }^{3}$, Nadav Davidovitch 3 , Jacob Moran-Gilad³, Amy Greer4, David Fisman5, John Brownstein 2, 6, Derek MacFadden2, 7}

${ }^{1}$ Public Health Agency of Sweden, Solna, Sweden, ${ }^{2}$ HealthMap, Boston Children's Hospital, Boston, Massachusetts, United States, ${ }^{3}$ Ben Gurion University, Beer- Sheva, Israel, ${ }^{4}$ University of Guelph, Guelph, Ontario, Canada, ${ }^{5}$ Dalla Lana School of Public Health, Toronto, Ontario, Canada,

${ }^{6}$ Harvard Medical School, Boston, Massachusetts, United States, ${ }^{7}$ Department of Medicine, University of Toronto, Toronto, Ontario, Canada

\section{Objective}

To develop, evaluate, and implement a universal online platform - termed OPEN Stewardship - to promote responsible antimicrobial prescribing (antimicrobial stewardship).

Introduction

Antibiotic resistance is a mounting public health threat calling for action on global, national and local levels. Antibiotic use has been a major driver of increasing rates of antibiotic resistance. This has given rise to the practice of antibiotic stewardship, which seeks to reduce unnecessary antibiotic use across different care settings. Antibiotic stewardship has been increasingly applied in hospital settings, but adoption has been slow in many ambulatory care settings including primary care of humans. Uptake of antibiotic stewardship in veterinary care has been similarly limited. Audit and feedback systems of antibiotic use coupled with patterns of antibiotic use and best practice guidelines have proven useful in outpatient settings, but scaleup is limited by heterogeneous systems of care and limited resources.

\section{Methods}

A multi-sectoral team with partners from Canada, Israel and Sweden is developing a web-based platform for administering antibiotic stewardship across multiple care settings and sectors, for human and animal prescribers. There are several interventions which support behaviour change and can be applied to antibiotic stewardship programs. Systematic reviews have found beneficial effects of numerous behaviour change interventions for optimizing clinical practice such as computerized reminders [1], opinion leaders as champions for change [2], and audit and feedback [3]. A recent Cochrane review [4] found that interventions to enable correct use of antibiotics improved policy compliance, and that enabling interventions that included feedback were more likely to be effective. We will use antibiotic prescribing benchmarking, focused guidelines, and local patterns of antibiotic resistance as key components that can be deployed as feedback through this antibiotic stewardship platform. The OPEN Stewardship platform will be hosted on an AWS cloud-based server using industry standard encryption. The platform will function with a central administrator who will enroll and deliver feedback to participating prescribers. This platform will be evaluated prospectively in two countries (Canada and Israel) to evaluate user experience of the feedback as well as impact on antimicrobial prescribing. The evaluation will include prescribers from both human and animal health. After the prospective evaluation, the platform will be made available online for broad multi-sectoral use. 
Results

We have designed the interface for a web-based platform for antibiotic stewardship which will be used in a multinational prospective primary care stewardship intervention in 2019 and 2020 and subsequently rolled out for broad public use (www.openasp.org). The platform layout can be seen in Figure 1. Data capture for aggregate prescriber level antibiotic use and local guidelines will be possible through both a manual graphical user interface and a dataset template upload. Antibiotic resistance data will be pulled from a companion database (www.resistanceopen.org). Administrators will be able to generate unique feedback forms containing visualizations and snapshots from antibiotic use, guidelines, and antibiotic resistance data (Figure 2). These can then be delivered by email on an individual or scheduled basis for one or multiple prescribers simultaneously. Participating prescribers will also have the option to login to view their own profile and browse antibiotic use, resistance and guidelines.

\section{Conclusions}

Antibiotic stewardship needs to be adopted in a fashion that is country and context specific and not administered from the top down. With our approach we seek to empower groups from any country or care setting to provide regional and tailored stewardship feedback through an open interface. We have here demonstrated the design of an web-based antibiotic stewardship platform which will be evaluated prospectively and subsequently made available for open and broad multi-sectoral use - in keeping with a One Health approach.

\section{Acknowledgement}

We would like to thank funding sources including: the Joint Programming Initiative in Antimicrobial Resistance, The Canadian Institutes for Health Research, The Swedish Research Council, and the Israel Ministry of Health.

\section{References}

1. Shojania KG, Jennings A, Mayhew A, Ramsay CR, Eccles MP, et al. 2009. The effects of on-screen, point of care computer reminders on processes and outcomes of care. Cochrane Database Syst Rev. (3), CD001096. PubMed

2. Flodgren G, Eccles MP, Shepperd S, Scott A, Parmelli E, et al. 2011. An overview of reviews evaluating the effectiveness of financial incentives in changing healthcare professional behaviours and patient outcomes. Cochrane Database Syst Rev. (7), CD009255. PubMed

3. Ivers N, Jamtvedt G, Flottorp S, Young JM, Odgaard-Jensen J, et al. 2012. Audit and feedback: effects on professional practice and healthcare outcomes. Cochrane Database Syst Rev. (6), CD000259. $\underline{\text { PubMed }}$

4. Davey P, Brown E, Charani E, Fenelon L, Gould IM, et al. 2013. Interventions to improve antibiotic prescribing practices for hospital inpatients. Cochrane Database Syst Rev. (4), CD003543. PubMed 

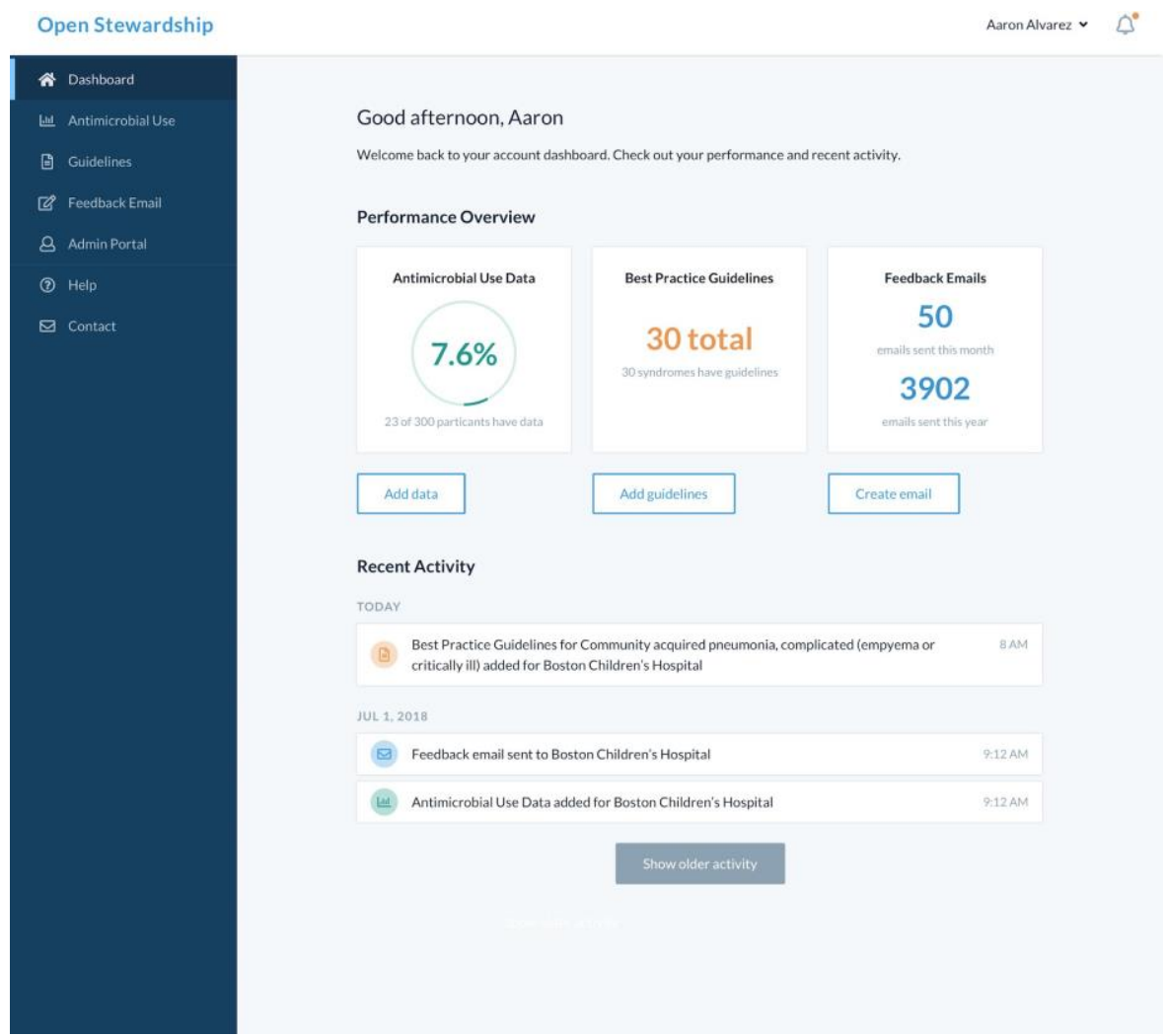

Figure 1: The platform interface.

Open Stewardship

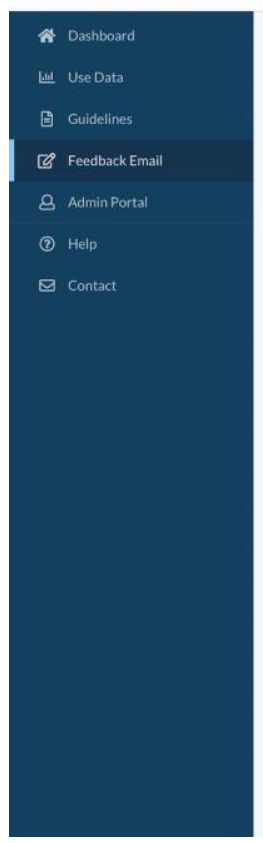

Aaron Alvarez $\sim$

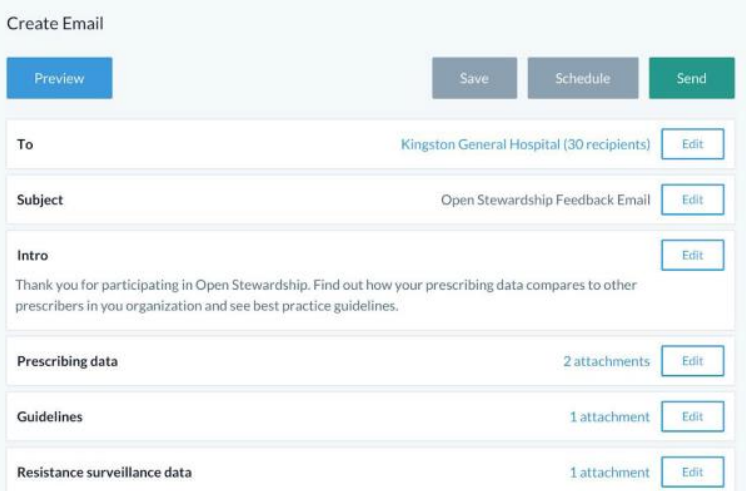

Figure 2: Generation of the feedback form/e-mail. 